\title{
Transaminase [alanine aminotransferase (ALT) and aspartate aminotransferase (AST)] Activity of HIVFemale Patients on Drugs and Female Patients Not on Drugs
}

\author{
ODIBA, Arome Solomon ${ }^{1}$, ONOSAKPONOMEIruoghene ${ }^{2}$, IROHA, \\ Okechukwu Kalu$^{2}$, UKEGBU, Chimere Young ${ }^{2}$, OMEJE, Kingsley Ozioma ${ }^{2}$ \\ ${ }^{1}$ Department of Biochemistry, University of Jos, Plateau State, Nigeria \\ ${ }^{2}$ Department of Biochemistry, University of Nigeria, Enugu State Nigeria
}

\begin{abstract}
Elevated serum transaminase [alanine aminotransferase (ALT) and aspartate aminotransferase $(A S T)]$ levels have been reported in human immunodeficiency virus (HIV)-infected patients on combination antiretroviral therapy (cART). 100 female patients referred to Virology department of the Jos University teaching Hospital (JUTH), were randomly selected by their physicians on the basis of the following criteria: documented HIV infection, no alcohol abuse $(\leq 20 \mathrm{~g}$ alcohol daily and no history of chronic alcohol consumption), no history of hereditary and autoimmune liver disease, no evidence of hemochromatosis. Patients with any contraindication for liver biopsy were excluded. Data on demographics, drug or alcohol use, and history of liver diseases were obtained at first visit. Subjects were then classified into control (30), HIV-infected; not on drugs (35) HIV-infected; on drugs (35); a month-by-month documentation of the prescribed drug combination for patients who started medication, provided an avenue for calculating each patient's individual cumulative drug exposure. The test for HIV antibodies, HBsAg were carried out on day one (at first visit) serum ALT and serum AST were determined three (3) months after detection (day 90; after HIV antigen confirmatory test). Thirty-five (35) patients started medication at day one and 35 decided not to start medication until after confirmatory test (three months later).The serum ALT levels of the HIV infected patients on drugs (M=43.94, $S D=4.30)$ was significantly higher than the serum ALT levels of the control group $(M=29.17, S D=6.17), t(63)$ $=10.72, p<.0001$ at $p<.005$ confidence level. In a similar trend, the serum ALT levels of the HIV infected patients not on drugs $(M=41.17, S D=2.06)$ was significantly higher than the serum ALT levels of the control group $(M=29.17, S D=6.17), t(63)=10.05, p<.0001$ at $p<.005$ confidence level. On comparison, the serum ALT levels of the HIV infected patients on drugs $(M=43.94, S D=4.30)$ was significantly higher than the serum ALT levels of the HIV infected patients not on drugs $(M=41.17, S D=2.06), t(68)=3.44, p<.0001$ at $p<.005$ confidence level.

The serum AST levels of the HIV infected patients on drugs $(M=44.54, S D=4.85)$ was significantly higher than the serum AST levels of the control group $(M=32.23, S D=5.93), t(63)=9.21, p<.0001$ at $p<.005$ confidence level. In a similar manner, the serum AST levels of the HIV infected patients not on drugs (M=39.91, $S D=1.67)$ was significantly higher than the serum AST levels of the control group $(M=32.23, S D=5.93), t(63)$ $=7.33, p<.0001$ at $p<.005$ confidence level. On comparison, the serum AST levels of the HIV infected patients on drugs $(M=44.54, S D=4.85)$ was significantly higher than the serum AST levels of the HIV infected patients not on drugs $(M=39.91, S D=1.67), t(68)=5.34, p<.0001$ at $p<.005$ confidence level. A correlation plot between ALT and AST levels showed a non-significant correlation between these transaminases in the three groups. Control group $(r=0.098, p<0.05)$, HIV (+ve), and HBV (-ve) patients on drugs $(r=-0.038, p<0.05)$, $H I V(+v e)$, and $H B V(-v e)$ patients not on drugs $(r=0.201, p<0.05)$.It has been observed from the study that a significant elevation of liver transaminase levels (ALT and AST) is a serious risk factor associated with management of HIV management with drugs.
\end{abstract}

Keywords: alanine transaminase, aspartate transaminase, drugs, female, hepatitis B virus, human immune deficiency virus

\section{Introduction}

AIDS has been reported in every country and parts of Africa and Asia are especially devastated by it. It has been estimated that about 35-40 million individuals are currently living with the virus [1]. HIV is an enveloped retrovirus containing single stranded RNA, which is the etiologic agent of AIDS and an infection is initiated by binding of the virion envelope gp 120 to the CD4 receptor on the host cell [2]. The onset of AIDS is associated with acute depletion of CD4 T-helper cells, as manifested by immune-deficiency, susceptibility to opportunistic infection and other AIDS-defining illness [3]. The clinical manifestations of HIV/AIDS can be seen in three phases: the initial acute phase, the latent phase and the advanced stage [4]. It is no doubt that combined antiretroviral therapy has brought about a drastic decline in HIV-associated mortality. However, liver- 
related morbidity has become a major issue in the HIV-infected population who are on combined antiretroviral therapy [5], [6]. Likewise, strong evidence exists for an interrelation between hepatic steatosis and combined antiretroviral therapy exposure [5]. Over time it has been studied that the use of stavudine has been closely linked to hepatic steatosis [7], whereas most protease inhibitors (PI) have been associated with metabolic abnormalities, hyperlipidemia, and decreased insulin sensitivity with subsequent development of hepatic steatosis [8], [9]. Elevated serum transaminase [alanine aminotransferase (ALT) and aspartate aminotransferase (AST)] levels have been reported in human immunodeficiency virus (HIV)-infected patients on combination antiretroviral therapy (cART) [10]. Human immunodeficiency virus-1 (HIV) is transmitted through sexual and percutaneous routes [11]. Management of HIV is complicated not only by the differences in natural history but also by other issues such as the activity of several drugs against the virus and development of drug-resistant HIV variants. The course of acute HIV may be modified in the presence of HBV infection, with a lower incidence of icteric illness and lower rates of spontaneous clearance of HBV. Persons with HIV and chronic HBV coinfection have higher levels of HBV DNA and lower rates of clearance of the hepatitis B antigen (HBeAg) [12], [13]. Liver disease has emerged as a leading cause of morbidity and mortality in HIV-infected individuals. The management of HIV infection iscomplicated by the dual activity of several nucleoside analogs. HIV increases the risk of cirrhosis and end-stage liver disease. A transaminase is a type of enzyme whose activity is frequently measured, as part of a standard series of tests, to determine liver function. There are a number of different types of transaminases, but the two commonly measured medically are alanine transaminase (ALT) and aspartate transaminase (AST). ALT is primarily localized to the liver and is considered a more specific test for liver damage. Elevated serum transaminase levels [alanine aminotransferase (ALT) and aspartate aminotransferase (AST)] in human immunodeficiency virus infected patients is an indication of a threat to the body system. Damages done on the body system as a result of infectious disease do reflect in the activities of liver transaminases. Liver damage is associated with chronic high serum transaminases in human immunodeficiency virus (HIV) infected patients under combined antiretroviral therapy. HIV infection patients portray more liver disease than those with HBV infection alone [14]. However, persons with HIV and HBV co-infection have a significant increase in liver-related mortality, compared to those who are infected with HIV only [15]. Also, HIV-infected patients co-infected with HBV have an increased risk for antiretroviral therapy-related hepatotoxicity since the exposure to these nucleoside analogues used in managing both infections is more intensive [16].There are many agents used to treat HIV infection, but the activity of these agents against HIV ranges from partial activity to high activity [17]. Antiretroviral agents that have strong activity against HIV include: tenofovir, emtricitabine, lamivudine amongst others. Most HIV medications contains one or more highly-active agents (e.g., lamivudine [3TC], emtricitabine [FTC], tenofovir [TDF]), that burden the liver with the duty of metabolizing these drugs with detrimental, damaging effect on the liver in addition to their therapeutic effect. In co-infected patients, IFN has been associated with lower rates of HIV treatment success and increased toxicity [18]. Although entecavir initially was thought to have no anti-HIV activity, it has been demonstrated to select for the M184V mutation.Therefore, ordering for a laboratory investigation on liver transaminases profile is a test to assess the level of damage.

The objectives of this study were to evaluate the liver transaminase levels as a direct index of hepatocellular damage, resulting from medications in the form of drugs use to manage HIV infection.

\section{Patients and Methods}

For this study 100 female patients referred to Virology department of the Jos University teaching Hospital (JUTH), were randomly selected by their physicians on the basis of the following criteria: documented HIV infection, no alcohol abuse ( $\leq 20 \mathrm{~g}$ alcohol daily and no history of chronic alcohol consumption), no history of hereditary and autoimmune liver disease, no evidence of hemochromatosis. Patients with any contraindication for liver biopsy were excluded. Data on demographics, drug or alcohol use, and history of liver diseases were obtained at first visit. Subjects were then classified into control (30), HIV-infected; on drugs (35) HIV-infected; not on drugs (35).A month-by-month documentation of the prescribed drug combination allowed a calculation of each patient's individual cumulative drug exposure. The test for HIV antibodies, HBsAg were carried out on day one (at first visit) serum ALT and serum AST were determined three (3) months after detection (day 90; after HIV antigen confirmatory test). Thirty-five (35) patients started medication at day one and 35 decided not to start medication until after confirmatory test (three months later). Both the HIV antibodies, HBsAg assessment was carried out using Fortress HIV 1\&2 STRIP and Fortress HbsAg ELISA test kit respectively. Serum ALT and serum AST were carried out spectrophotometrically.

\subsection{Parameters}

The biochemical parameters for laboratory assessment included HIV antibodies, HBsAg, serum ALT and serum AST. 


\subsection{Statistical analysis.}

Variables with a normal distribution were expressed as mean and standard deviation as well as comparison using T test. All measured variables were considered in analysis and also a correlation analysis between the ALT and AST was run. Analyses were performed using IBM SPSS package version 20.

\section{Results}

Table 1: ALT and AST levels of control group [HIV (-ve), HBV (-ve)]

\begin{tabular}{|c|c|c|c|c|}
\hline S/N of patients & HIV Status & HBV Status & ALT (Iu/L) & AST/ (Iu /L) \\
\hline 1 & - & - & 38 & 38 \\
\hline 2 & - & - & 37 & 37 \\
\hline 3 & - & - & 40 & 40 \\
\hline 4 & - & - & 22 & 36 \\
\hline 5 & - & - & 19 & 37 \\
\hline 6 & - & - & 17 & 36 \\
\hline 7 & - & - & 33 & 36 \\
\hline 8 & - & - & 24 & 24 \\
\hline 9 & - & - & 23 & 26 \\
\hline 10 & - & - & 22 & 33 \\
\hline 11 & - & - & 27 & 37 \\
\hline 12 & - & - & 36 & 40 \\
\hline 13 & - & - & 37 & 39 \\
\hline 14 & - & - & 36 & 18 \\
\hline 15 & - & - & 36 & 22 \\
\hline 16 & - & - & 26 & 34 \\
\hline 17 & - & - & 31 & 33 \\
\hline 18 & - & - & 34 & 33 \\
\hline 19 & - & - & 25 & 24 \\
\hline 20 & - & - & 24 & 24 \\
\hline 21 & - & - & 26 & 35 \\
\hline 22 & - & - & 23 & 39 \\
\hline 23 & - & - & 33 & 29 \\
\hline 24 & - & - & 33 & 26 \\
\hline 25 & - & - & 40 & 37 \\
\hline 26 & - & - & 22 & 34 \\
\hline 27 & - & - & 23 & 28 \\
\hline 28 & - & - & 31 & 30 \\
\hline 29 & - & - & 33 & 30 \\
\hline 30 & - & - & 24 & 32 \\
\hline
\end{tabular}

Table 2: ALT and AST levels of HIV (+ve), and HBV (-ve) patients on drugs

\begin{tabular}{|c|c|c|c|c|}
\hline S/N of patients & HIV Status & HBV Status & ALT (Iu/L) & AST $\quad(\mathrm{Iu} / \mathrm{L})$ \\
\hline 031 & + & - & 42 & 43 \\
\hline 032 & + & - & 42 & 41 \\
\hline 033 & + & - & 43 & 42 \\
\hline 034 & + & - & 45 & 54 \\
\hline 035 & + & - & 51 & 53 \\
\hline 036 & + & - & 44 & 49 \\
\hline 037 & + & - & 49 & 49 \\
\hline 038 & + & - & 45 & 40 \\
\hline 039 & + & - & 47 & 40 \\
\hline 040 & + & - & 39 & 44 \\
\hline 041 & + & - & 44 & 43 \\
\hline 042 & + & - & 40 & 38 \\
\hline 043 & + & - & 43 & 39 \\
\hline 044 & + & - & 43 & 39 \\
\hline 045 & + & - & 43 & 49 \\
\hline 046 & + & - & 44 & 45 \\
\hline 047 & + & - & 48 & 47 \\
\hline 048 & + & - & 42 & 48 \\
\hline 049 & + & - & 44 & 55 \\
\hline 050 & + & - & 39 & 43 \\
\hline 051 & + & - & 39 & 45 \\
\hline 052 & + & - & 49 & 40 \\
\hline 053 & + & - & 51 & 36 \\
\hline 054 & + & - & 48 & 43 \\
\hline 055 & + & - & 38 & 55 \\
\hline
\end{tabular}


Transaminase [alanine aminotransferase (ALT) and aspartate aminotransferase (AST)] Activity of

\begin{tabular}{|l|l|l|l|l|}
\hline 056 & + & - & 39 & 42 \\
\hline 057 & + & - & 40 & 43 \\
\hline 058 & + & - & 40 & 44 \\
\hline 059 & + & - & 44 & 43 \\
\hline 060 & + & - & 43 & 47 \\
\hline 061 & + & - & 43 & 49 \\
\hline 062 & + & - & 37 & 44 \\
\hline 063 & + & - & 55 & 41 \\
\hline 064 & + & - & 52 & 46 \\
\hline 065 & + & - & 43 & 40 \\
\hline
\end{tabular}

Table 2: ALT and AST levels of HIV (+ve), and HBV (-ve) patients not on drugs

\begin{tabular}{|c|c|c|c|c|}
\hline S/N of patients & HIV Status & HBV Status & ALT (Iu/L) & AST $\quad(\mathrm{Iu} / \mathrm{L})$ \\
\hline 066 & + & - & 38 & 40 \\
\hline 067 & + & - & 39 & 39 \\
\hline 068 & + & - & 38 & 38 \\
\hline 069 & + & - & 40 & 38 \\
\hline 070 & + & - & 40 & 38 \\
\hline 071 & + & - & 40 & 40 \\
\hline 072 & + & - & 45 & 40 \\
\hline 073 & + & - & 42 & 39 \\
\hline 074 & + & - & 42 & 39 \\
\hline 075 & + & - & 41 & 42 \\
\hline 076 & + & - & 40 & 40 \\
\hline 077 & + & - & 39 & 39 \\
\hline 078 & + & - & 38 & 38 \\
\hline 079 & + & - & 40 & 40 \\
\hline 080 & + & - & 40 & 43 \\
\hline 081 & + & - & 43 & 44 \\
\hline 082 & + & - & 42 & 38 \\
\hline 083 & + & - & 43 & 40 \\
\hline 084 & + & - & 44 & 40 \\
\hline 085 & + & - & 43 & 44 \\
\hline 086 & + & - & 43 & 40 \\
\hline 087 & + & - & 41 & 39 \\
\hline 088 & + & - & 39 & 40 \\
\hline 089 & + & - & 40 & 39 \\
\hline 090 & + & - & 38 & 42 \\
\hline 091 & + & - & 42 & 41 \\
\hline 092 & + & - & 44 & 40 \\
\hline 093 & + & - & 44 & 39 \\
\hline 094 & + & - & 45 & 38 \\
\hline 095 & + & - & 42 & 40 \\
\hline 096 & + & - & 40 & 40 \\
\hline 097 & + & - & 41 & 39 \\
\hline 098 & + & - & 41 & 38 \\
\hline 099 & + & - & 40 & 40 \\
\hline 100 & + & - & 44 & 43 \\
\hline
\end{tabular}

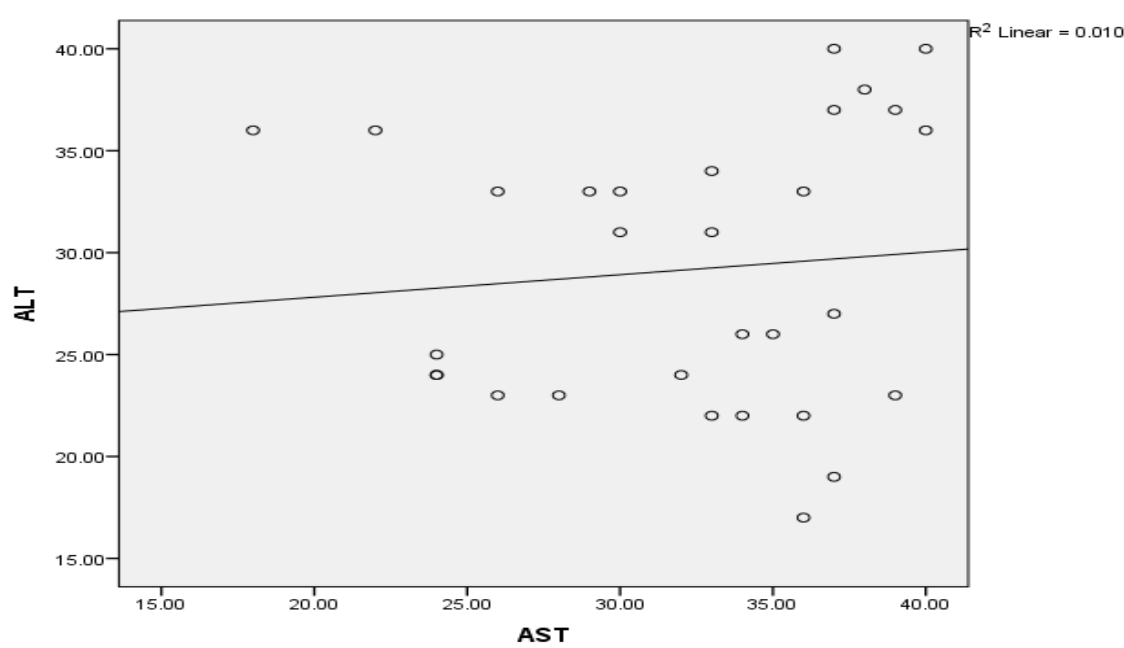

Figure 1: a correlation plot between ALT and AST levels of the control group 


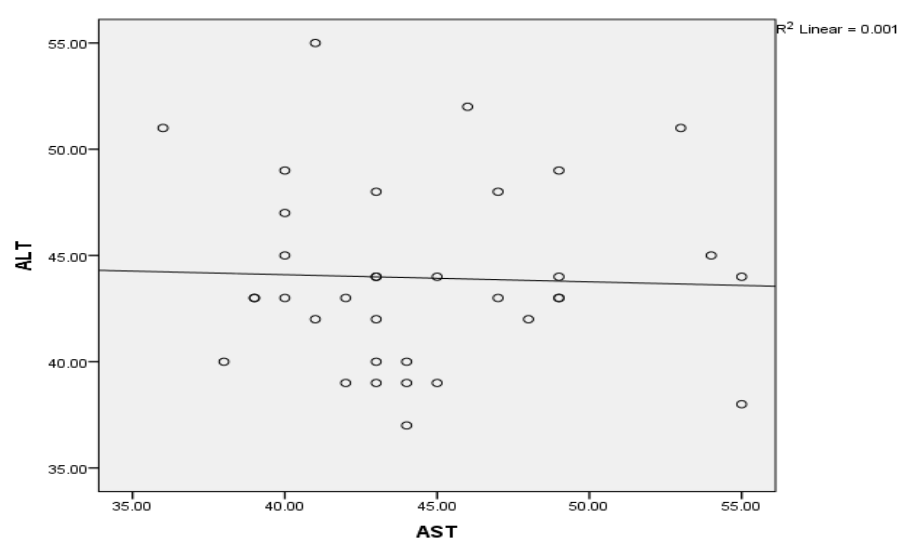

Figure 2: a correlation plot between ALT and AST levels of HIV (+ve), and HBV (-ve) patients on drugs

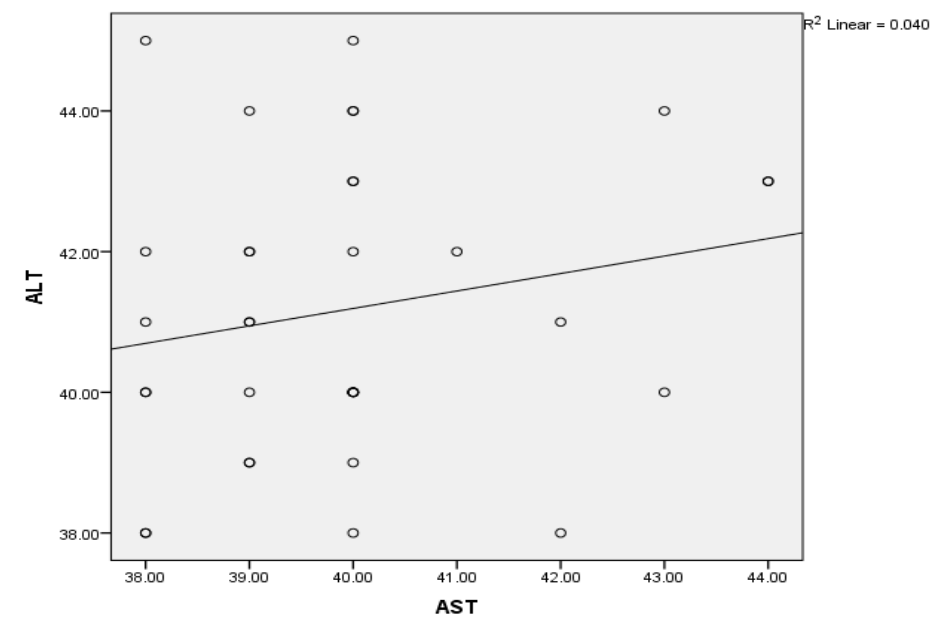

Figure 3: a correlation plot between ALT and AST levels of HIV (+ve), and HBV (-ve) patients not on drugs

\section{Discussion}

The serum ALT levels of the HIV infected patients on drugs $(\mathrm{M}=43.94, \mathrm{SD}=4.30)$ was significantly higher than the serum ALT levels of the control group $(\mathrm{M}=29.17, \mathrm{SD}=6.17), t(63)=10.72, p<.0001$ at $p<.005$ confidence level. In a similar trend, the serum ALT levels of the HIV infected patients not on drugs $(\mathrm{M}=41.17$, $\mathrm{SD}=2.06)$ was significantly higher than the serum ALT levels of the control group $(\mathrm{M}=29.17, \mathrm{SD}=6.17), t(63)$ $=10.05, p<.0001$ at $p<.005$ confidence level. On comparison, the serum ALT levels of the HIV infected patients on drugs $(M=43.94, S D=4.30)$ was significantly higher than the serum ALT levels of the HIV infected patients not on drugs $(\mathrm{M}=41.17, \mathrm{SD}=2.06), t(68)=3.44, p<.0001$ at $p<.005$ confidence level.

The serum AST levels of the HIV infected patients on drugs $(\mathrm{M}=44.54, \mathrm{SD}=4.85)$ was significantly higher than the serum AST levels of the control group $(\mathrm{M}=32.23, \mathrm{SD}=5.93), t(63)=9.21, p<.0001$ at $p<.005$ confidence level. In a similar manner, the serum AST levels of the HIV infected patients not on drugs $(\mathrm{M}=39.91, \mathrm{SD}=1.67)$ was significantly higher than the serum AST levels of the control group $(\mathrm{M}=32.23, \mathrm{SD}=5.93), t(63)=7.33, p<$ .0001 at $p<.005$ confidence level. On comparison, the serum AST levels of the HIV infected patients on drugs $(\mathrm{M}=44.54, \mathrm{SD}=4.85)$ was significantly higher than the serum AST levels of the HIV infected patients not on drugs $(\mathrm{M}=39.91, \mathrm{SD}=1.67), t(68)=5.34, p<.0001$ at $p<.005$ confidence level.

From the forgoing, flares in serum ALT and AST could result from many factors, which could be a direct extrapolation of the damaging effect. In patients with chronic HIV-infection who experience HIV suppression while receiving ART, flares have been reported following the discontinuation of lamivudine [19], emtricitabine [20], or tenofovir [21] treatment or with the emergence of lamivudine resistance [21].For HIVinfected patients, flares have been reported when they start receiving combination antiretroviral therapy, and the flares may be attributed to drug related hepatotoxicity [22].Also flares have been recorded after the cessation of lamivudine, emtricitabine, adefovir, or tenofovir [19], [23], [24]. It has been observed from this study that a significant elevation of liver transaminase levels (ALT and AST) is a serious risk factor associated with management of HIV management with drugs. This is a reasonable explanation of the liver damaging potential of drugs use in the management of HIV. A correlation plot between ALT and AST levels showed a non-significant 
correlation between these transaminases in the three groups. Control group $(r=0.098, p<0.05)$, HIV (+ve), and HBV (-ve) patients on drugs $(r=-0.038, p<0.05)$, HIV (+ve), and HBV (-ve) patients not on drugs $(r=0.201$, p $<0.05$ ). This shows that it's not a must that errors will occur in processes that has to do with the both enzymes. This explains the fact that they both have similar but independent metabolic routes.

\section{Conclusion}

In addition to the therapeutic effect of drugs used in the management of HIV, they have some damaging effect on the system, especially on the liver which is burdened with the duty of metabolizing these drugs. HIV infection contributes significantly to hepatic morbidity and mortality. Other factors may also cause the levels of these aminotransferases to elevate. Therefore, such other factors should be investigated alongside HIV infection investigation. It is paramount to know that drugs with minimal damaging effect on the liver, yet effective, should be used to manage such patients.

\section{Acknowledgements}

We wish to sincerely appreciate the virology department of the Jos University Teaching Hospital (JUTH) for making their patients as well as equipment available for this study.

\section{References}

[1] F. C. Bonnet, D. Lewden, L. May, Heripret, E. Jougla, Malignancy-related causes of death in human immunodeficiency virusinfected patients in the era of highly active antiretroviral therapy, Cancer, 101, 2004, 317-324.

[2] C. Feldman, Pneumonia associated with HIV infection, Current Opinion on Infectious Diseases, 18, 2005, $165-170$.

[3] J. Palefsky, Human papilloma virus infection in HIV-infected persons, Top HIV Med., 15, 2007, 130-133.

[4] J. Watson, Scientists, activists sue South Africa`s AIDS denialists,Nat. Med., 12, 2006, 6-6.

[5] K. A. Gebo, M. Diener-West, R. D. Moore, Hospitalization rates differ by hepatitis C status in an urban HIV cohort, Journal of Acquired Immune Deficiency Syndrome,34, 2003,165-173.

[6] I. Bica, B. McGovern, R. Dhar, D. Stone, K. McGowan, R. Scheib, Increasing mortality due to end-stage liver disease in patients with human immunodeficiency virus infection, Clinical Infectious Diseases, 32, 2001, 492-497.

[7] M. S. Sulkowski, S. H. Mehta, M. Torbenson, N. H. Afdhal, L. Mirel, R. D. Moore, Hepatic steatosis and antiretroviral drug use among adults coinfected with HIV and hepatitis C virus, AIDS, 19, 2005, 585-592.

[8] A. Lonardo, L. E. Adinolfi, P. Loria, N. Carulli, G. Ruggiero, C. P. Day, Steatosis and hepatitis C virus: mechanisms and significance for hepatic and extrahepatic disease, Gastroenterology, 126, 2004, 586-597.

[9] S. H. Mehta, R. D. Moore, D. L. Thomas, R. E. Chaisson, M. S. Sulkowski, The effect of HAART and HCV infection on the development of hyperglycemia among HIV-infected persons, Journal of Acquired Immune Deficiency Syndrome, 33, 2003, 577584 .

[10] M. Nu'ñez, Hepatotoxicity of antiretrovirals: incidence, mechanisms and management, Journal of Hepatology, 44, 2006, $132-139$.

[11] B. H. McGovern, The epidemiology, natural history and prevention of hepatitis B: implications of HIV co-infection, Antiviral Therapy, 12, 2007, H3-H13.

[12] C. L. Thio, Hepatitis B in the human immunodeficiency virus-infected patient: epidemiology, natural history, and treatment, Seminars on Liver Disease, 23(2), 2003, 125-136.

[13] L. Piroth, D. Sène, S. Pol, Epidemiology, diagnosis and treatment of chronic hepatitis B in HIV-infected patients (EPIB 2005 STUDY). AIDS, 21(10), 2007, 1323-3131.

[14] M. Puoti, C. Torti, R. Bruno, G. Filice, G. Carosi, Natural history of chronic hepatitis B in co-infected patients, Journal of Hepatology, 44, 2006, S65-70.

[15] C. L. Thio, E. C. Seaberg, R. Skolasky, HIV-1, hepatitis B virus, and risk of liver-related mortality in the Multicenter Cohort Study (MACS), Lancet, 360, 2002, 1921-1926.

[16] M. Savès, S. Vandentorren, V. Daucourt, Severe hepatic cytolysis: incidence and risk factors in patients treated by antiretroviral combinations. Aquitaine Cohort, France, 1996-1998. Groupe dEpidémiologie Clinique de Sida en Aquitaine (GECSA), AIDS, 13(17), 1999, F115-121.

[17] J. E. Kaplan, C. Benson, K. K. Holmes, Guidelines for prevention and treatment of opportunistic infections in HIV-infected adults and adolescents: recommendations from CDC, the National Institutes of Health, and the HIV Medicine Association of the Infectious Diseases Society of America, MMWR Recommendations and Reports, 58(RR-4), 2009, 1-207.

[18] V. Di Martino, T. Thevenot, J. F. Colin, Influence of HIV infection on the response to interferon therapy and the long-term outcome of chronic hepatitis B, Gastroenterology, 123(6) 2002, 1812-1822.

[19] M. Bessesen, D. Ives, L. Condreay, S. Lawrence, K. Sherman, Chronic active hepatitis B exacerbations in human immunodeficiency virus- infected patients following development of resistance, Clinical Infectious Diseases, 28, 1999, 1032-1035.

[20] E. Mondou, J. Sorbel, J. Anderson, H. Mommeja-Marin, A. Rigney, F. Rousseau, Posttreatment exacerbation of hepatitis B virus (HBV) infection in long-term HBV trials of emtricitabine, Clinical Infectious Diseases, 41, 2005, e45-47.

[21] L. E. Chapman, E.E. Sullivent, L. A. Grohskopf, E. M. Beltrami, J. F. Perz, K. Kretsinger, Recommendations for postexposure interventions to prevent infection with hepatitis B virus, hepatitis C virus, or human immunodeficiency virus, and tetanus in persons wounded during bombings and other mass-casualty events-United States, 2008: recommendations of the Centers for Disease Control and Prevention (CDC), MMWR Recommendations and Reports57(RR-6), 2008, 1-21.

[22] V. Soriano, J. Miro, J. Garcia-Samaniego, Consensus conference on chronic viral hepatitis and HIV infection: updated Spanish recommendations, Journal of Viral Hepatology, 11, 2004, 2-17

[23] Gilead Pharmaceuticals. Adefovir full prescribing information. Available at: http://www.gilead.com, Accessed 20 April 2006.

[24] Gilead Pharmaceuticals. Tenofovir full prescribing information. Available at: http:// www.gilead.com, Accessed 20 April 2006 